\title{
Compressive sensing based joint frequency offset and channel estimation for OFDM
}

\author{
Rıfat Volkan Şenyuva ${ }^{*}$, Güneş Karabulut Kurt ${ }^{2}$ and Emin Anarim ${ }^{1}$
}

\begin{abstract}
We consider joint estimation of carrier frequency offset (CFO) and channel impulse response (CIR) for orthogonal frequency division multiplexing (OFDM) with pilot symbols. A new method based on compressed sensing is proposed. It has been shown that the CIR can be represented as a 1-block sparse signal by using a dictionary constructed by concatenating subspaces of CFO values taken from a search space. Recovery of both CFO and CIR is accomplished by the block orthogonal matching pursuit algorithm. The proposed method uses only one OFDM training block and does not require any initialization. The performance of the proposed method is compared against the well-established pilot based estimators: Moose, Classen, the maximum likelihood estimator, and the p-algorithm. Numerical results show that the performance of the proposed method does not depend on the value of the CFO. We also give worst-case upper bounds for the mean squared error of the CIR estimate for a sparse multipath channel.
\end{abstract}

Keywords: Carrier frequency offset, Channel estimation, Compressive sensing, Block-sparsity, OFDM

\section{Introduction}

Orthogonal frequency division multiplexing (OFDM) has become a standard multi-carrier modulation technique for broadband wireless communication networks due to its resistance to interblock interference (IBI) caused by frequency-selective multipath fading channels. The success of OFDM systems relies on the orthogonality property of its chosen subcarriers which transforms the frequency selective channel into a set of frequency flat fading channels simplifying the equalization task. However, it is known that OFDM is very sensitive to both frequency synchronization and channel estimation errors. The oscillator mismatches and/or Doppler shifts introduce carrier frequency offset (CFO) which destroys the orthogonality amongst the subcarriers. As a result, the signal constellation is rotated and inter-carrier interference (ICI) occurs. The quality of the channel estimates, which is vital for coherent data detection, is also affected negatively under CFO. All of these effects reduce the effective signal-to-noise ratio (SNR) at the receiver and degrade the

\footnotetext{
*Correspondence: volkan.senyuva@boun.edu.tr

${ }^{1}$ Department of Electrical and Electronics Engineering, Bogazici University,

Bebek, Istanbul, Turkey

Full list of author information is available at the end of the article
}

performance of the OFDM system. Thus, accurate CFO and channel estimation is essential for exploiting the full potential of OFDM systems.

CFO and channel estimation can be done separately by first estimating the CFO and then performing channel estimation in the second step. Both training symbolbased and blind CFO estimators have been proposed in the literature [1-5]. The estimator proposed by Moose uses repeated data symbols [1]. Classen's method inserts pilot subcarriers in OFDM blocks for CFO estimation and assume that the channel does not change for two consecutive OFDM blocks [2]. Both methods assume a sufficiently small CFO and also a not high SNR so that the ICI is much smaller than the noise and can be ignored. They are sensitive to the value of the CFO and are valid for small CFO values. The blind estimator developed by Beek exploits the redundancy in the cyclic prefix (CP) [3]. A blind estimator based on MUSIC subspace method is developed in [4] using the virtual subcarriers (VC). The p-algorithm in [5] considers both $\mathrm{VC}$ and pilot carriers in each OFDM block but assumes that the channel remains constant for two OFDM blocks like the Classen method. Channel estimation can be

\section{Springer}

(c) 2016 Senyuva et al. Open Access This article is distributed under the terms of the Creative Commons Attribution 4.0 International License (http://creativecommons.org/licenses/by/4.0/), which permits unrestricted use, distribution, and reproduction in any medium, provided you give appropriate credit to the original author(s) and the source, provide a link to the Creative Commons license, and indicate if changes were made. 
performed after applying these well-established CFO synchronization methods. However, since perfect synchronization is not possible, the residual CFO will degrade the performance of the channel estimate significantly. Thus, better performance can be obtained when CFO and channel are estimated jointly. There exists a number of joint CFO and channel estimators that use pilot symbols [6-9]. Both [6] and [7] use the framework of the expectation-maximization (EM) algorithm. While [6] directly computes the channel parameters, [7] estimates the parameters of a basis expansion-based parametric channel model assuming the channel delays are known. The initialization of [6] requires a coarse CFO estimate such as provided by the Beek's method [3]. A joint maximum likelihood estimate (MLE) of CFO and channel impulse response (CIR) using a training symbol is given in [8]. The estimator in [9] is an approximate MLE since the received signal samples of the OFDM system are assumed to be Gaussian. Also, both [6] and [9] require the secondorder statistics of the channel and noise. For [9], an initial estimate of both the channel and CFO is necessary to initialize the joint MLE.

A joint estimator of CFO and CIR using the compressive sensing (CS) framework is proposed in this paper. The framework of CS coined in [10] deals with the recovery of an unknown signal from an underdetermined system of linear equations. By exploiting the key property that the unknown signal is sparse, only a few entries of the signal are nonzero, the perfect reconstruction of the unknown signal is possible even if the system is underdetermined. Sparse signals may have additional structure in the form of nonzero coefficients occurring in clusters (such as in $[11,12]$ ). Such signals are referred to as blocksparse signals $[11,12]$. A similar structure can be created by concatenating dictionaries of OFDM training symbols perturbed by CFO values taken from a search space and with such a structure the CIR can be represented as a 1-block sparse signal. The proposed framework allows solving for both CFO and CIR simultaneously by using the CS recovery algorithms for block-sparse signals. To the best of our knowledge, the proposed method is the first CS-based approach towards the joint estimation of both CFO and CIR for an OFDM system. There exist numerous implementations of the CS-based block sparse signal recovery methods for the estimation of the CIR of the OFDM systems [13-15]. In these works, block sparsity is achieved by either assuming that the several channel instantiations are group-sparse, locations of the nonzero channel coefficients are same, [15] or concatenating multiple CIRs of different antennas with common support in a block sparse structure $[13,14]$. However, frequency offset is not considered in these papers, which assume perfect synchronization for the OFDM system and so only CIR can be estimated using these methods. The block orthogonal matching pursuit (BOMP) algorithm which has a computational complexity of $\mathrm{O}\left(d N N_{g}\right), d$ is the length of the search space, $N$ is the number of subcarriers, and $N_{g}$ is cyclic prefix length, is used as the recovery algorithm. The MLE makes use of an FFT-based search of complexity $d\left(5 \beta \log _{2} d+1\right)$ in flops, where $\beta$ denotes the saving for skipping operations on the zeros in the FFT. However, the input of this implementation requires a matrix inversion step which cannot always be precomputed if the channel and noise statistics are not available [9]. As a result, the complexity of the MLE is determined by the most costly computation step that is the matrix inversion $\mathrm{O}\left(N^{3}\right)$. The p-algorithm does not involve matrix inversion and can be implemented in two ways: FFT-based search and polynomial rooting [5]. The complexity of the polynomial rooting which is recommended for smaller $N$ and $N_{g}$ is approximated as $\mathrm{O}\left(\left(2 N+N_{g}\right)^{3}\right)$ [5].

The main contributions of our work are the following:

- The proposed method only needs one OFDM block of training symbols unlike Moose, Classen, and the p-algorithm. The use of multiple blocks makes the estimation susceptible to changes in the channel or the CFO. Also, the use of more blocks means an increase in pilot overhead.

- The proposed method does not require any initialization or the second-order statistics of the channel and noise unlike EM-based methods. Apriori knowledge about either the channel or the noise may not be available for every case.

- The performance of the proposed method does not depend on the value of the CFO.

- Our work makes use of the worst-case bounds of the perturbed CS recovery for sparse multipath channel estimation. These bounds provide a way to observe how the performance of the sparse channel estimation methods scales with the perturbation due to frequency offset.

\section{System model}

Information is transmitted in blocks of symbols in an OFDM system. An OFDM block consists of $N$ transmitted symbols $X_{m}[k], k=0, \ldots, N-1$, each chosen from a signal constellation. Time domain samples of the OFDM block is obtained after performing inverse discrete Fourier transform (IDFT) on the transmitted symbols

$$
x_{m}[n]=\frac{1}{N} \sum_{k=0}^{N-1} X_{m}[k] w_{N}^{k\left(n-N_{g}\right)}, n=N_{g}, \ldots, N_{t}-1,
$$

where $m$ denotes the OFDM block index, $w_{N}=e^{j 2 \pi / N}$, and $N_{t}$ is the total number of samples in a OFDM block 
including the CP samples, $N_{t}=N+N_{g}$. CP samples required to ensure that no IBI occurs and so the first $N_{g}$ samples of the OFDM block, $\left\{x_{m}[0], \ldots, x_{m}\left[N_{g}-1\right]\right\}$, are taken same as the last $N_{g}$ samples of the OFDM block $\left\{x_{m}\left[N_{t}-N_{g}\right], \ldots, x_{m}\left[N_{t}-1\right]\right\}$.

When the oscillator of the receiver is not perfectly matched to the carrier of the received signal, CFO denoted as $\Delta f$ occurs. CFO can be normalized as $\epsilon=\Delta f N T$ where $1 /(N T)$ is the subcarrier bandwidth and $T$ is the sampling period. The received signal after transmitting $x_{m}[n]$ through a channel $h_{m}[n]$ can be written in terms of the normalized CFO as

$$
y_{m}[n]=c_{m}(\epsilon, n)\left(h_{m}[n] * x_{m}[n]\right)+z_{m}[n],
$$

where $*$ is the convolution operator, i.e., $h_{m}[n] * x_{m}[n]=$ $\sum_{l=0}^{N_{g}} h_{m}[l] x_{m}[n-l]$, and $z_{m}$ is zero mean complex additive white Gaussian noise with variance $\sigma_{z}^{2}$, and

$$
c_{m}(\epsilon, n)=e^{j 2 \pi \epsilon n / N} e^{j 2 \pi \epsilon m\left(N_{t} / N\right)} .
$$

After performing discrete Fourier transform (DFT) on the received samples, frequency domain-received samples are given as

$$
Y_{m}[k]=C_{m}(\epsilon, k) *\left(H_{m}[k] X_{m}[k]\right)+Z_{m}[k],
$$

where $C_{m}(\epsilon, k), H_{m}[k]$ and $X_{m}[k]$ are DFT of $c_{m}(\epsilon, n)$, $h_{m}[n]$ and $x_{m}[n]$, respectively. $C_{m}(\epsilon, k)$ is expressed as

$$
\begin{aligned}
C_{m}(\epsilon, k)= & \left(\frac{\sin (\pi(\epsilon-k))}{N \sin (\pi(\epsilon-k) / N)} e^{j \pi(\epsilon-k) \frac{N-1}{N}}\right) \\
& e^{j\left(2 \pi \epsilon m\left(N_{t} / N\right)\right)} e^{j\left(2 \pi \epsilon\left(N_{g} / N\right)\right)} .
\end{aligned}
$$

When the relative frequency offset is greater than $|\epsilon| \geq$ 0.5 , the frequency offset introduces a cyclic shift of $Y_{m}[k]$ [16]. When $|\epsilon| \geq 0.5$, the integer frequency offset correction algorithms such as $[17,18]$ are employed. In this letter, the relative frequency offset is assumed to be $|\epsilon|<$ 0.5 . The received symbol at the $k$-th subcarrier can be written as

$$
Y_{m}[k]=C_{m}(\epsilon, 0) H_{m}[k] X_{m}[k]+I_{m}[k]+Z_{m}[k],
$$

where $I_{m}[k]$ is given as

$$
I_{m}[k]=\sum_{r=1}^{N-1} C_{m}(\epsilon, r) H_{m}[k-r] X_{m}[k-r] .
$$

As it is seen from (6), the received signal is affected by the CFO. While the magnitude of the signal $H_{m}[k] X_{m}[k]$ is attenuated by $\left|\frac{\sin (\pi \epsilon)}{N \sin (\pi \epsilon / N)}\right|$, its phase is increased by $\pi \epsilon(1-1 / N)+2 \pi \epsilon\left[m\left(N_{t} / N\right)+\left(N_{g} / N\right)\right]$. In addition to noise $Z_{m}[k]$, ICI denoted as $I_{m}[k]$ is added to the signal. We derive our proposed estimator by using the frequency domain model given in Eq. (6), as detailed below.

\section{Compressive sensing-based joint frequency offset and channel estimation}

Fixing the OFDM block index $m$, the DFT applied received signal samples can be represented in matrix form as

$$
\tilde{\mathbf{y}}=\mathbf{C}(\epsilon) \mathbf{X F}\left[\begin{array}{c}
h[0] \\
\vdots \\
h[N-1]
\end{array}\right]+\left[\begin{array}{c}
Z[0] \\
\vdots \\
Z[N-1]
\end{array}\right],
$$

where $\mathbf{F}$ denotes the $N$-point DFT matrix, $\tilde{\mathbf{y}}=$ $[Y[0] \cdots Y[N-1]]^{T}, \mathbf{X}=\operatorname{diag}\{X[0], \ldots, X[N-1]\}$, and $\mathrm{C}(\epsilon)$ is given as

$$
\mathbf{C}(\epsilon)=\left[\begin{array}{cccc}
C(0) & C(N-1) & \cdots & C(1) \\
C(1) & C(0) & \cdots & C(2) \\
\vdots & \ddots & \ddots & \vdots \\
C(N-1) & C(N-2) & \cdots & C(0)
\end{array}\right] .
$$

Assuming that channel length is smaller than the number of subcarriers $L \ll N$ and the number of cyclic prefix is chosen as $N_{g} \geq L$, the channel coefficient vector is zero-padded as $\mathbf{h}=\left[h[0] \cdots h\left[N_{g}-1\right], \mathbf{0}_{1 \times N-N_{g}}\right]^{T}$. The zeros can be removed by using the first $N_{g}$ columns of the $N$-point DFT matrix $\mathbf{F}$ that is $\mathbf{F}_{N_{g}}$

$$
\begin{aligned}
\tilde{\mathbf{y}} & =\mathbf{C}(\epsilon) \mathbf{X F}_{N_{g}}\left[\begin{array}{c}
h[0] \\
\vdots \\
h\left[N_{g}-1\right]
\end{array}\right]+\mathbf{z} \\
& =\tilde{\mathbf{A}} \mathbf{h}+\mathbf{z},
\end{aligned}
$$

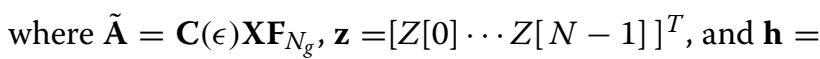
$\left[h[0] \cdots h\left[N_{g}-1\right]\right]^{T}$.

A search space for the fractional frequency offset can be created by a set of $d$ values $\left\{\tilde{\epsilon}_{0}, \tilde{\epsilon}_{1}, \ldots, \tilde{\epsilon}_{d-1}\right\} \in[-0.5,0.5]$. Each value $\tilde{\epsilon}_{i}$ corresponds to a $\mathbf{C}\left(\tilde{\epsilon}_{i}\right)$ and the received samples can be rewritten as

$$
\begin{aligned}
& \tilde{\mathbf{y}}=\left[\mathbf{C}\left(\tilde{\epsilon}_{0}\right) \mathbf{X F}_{N_{g}} \cdots \mathbf{C}\left(\tilde{\epsilon}_{d-1}\right) \mathbf{X F}_{N_{g}}\right] \tilde{\mathbf{h}}+\mathbf{z} \\
& =\mathbf{D} \tilde{\mathbf{h}}+\mathbf{z} \text {, }
\end{aligned}
$$

where the vector $\tilde{\mathbf{h}}$ is built by stacking $d$ blocks of $N_{g} \times$ 1 channel vectors, $\tilde{\mathbf{h}}=\left[\mathbf{h}_{0} \ldots \mathbf{h}_{d-1}\right]$, and the matrix $\mathbf{D}$ is constructed by concatenating $d$ matrices each corresponding to a $\mathbf{C}\left(\tilde{\epsilon}_{i}\right)$. Each block of $\tilde{\mathbf{h}}$ is represented as $\tilde{\mathbf{h}}[i]=\mathbf{h}_{i}=\left[h[0], \ldots, h\left[N_{g}-1\right]\right]^{T}$. In this representation, $\tilde{\mathbf{h}}$ can be considered as a 1-block sparse vector.

The problem of finding a sparse representation which uses the minimum number of blocks of $\mathbf{D}$ is given as the following optimization problem

$$
\begin{array}{ll}
\text { minimize } & \sum_{i=0}^{d-1} I\left(\|\tilde{\mathbf{h}}[i]\|_{q}\right) \\
\text { subject to } & \tilde{\mathbf{y}}=\mathbf{D} \tilde{\mathbf{h}},
\end{array}
$$


where $q \geq 0$ and $I(\cdot)$ is the indicator function which is zero when its argument is zero and is one otherwise. The indicator function counts the number of nonzero blocks of a solution. However, solving (12) is an NP hard problem since it involves searching over all choices of a few blocks of $\mathbf{D}[11,12]$. A number of algorithms such as Group Lasso [19], mixed $\ell_{2} / \ell_{1}$ program [20], block orthogonal matching pursuit (BOMP) algorithm [11], block sparse Bayesian learning [21], block iteratively reweighted least squares (BIRLS) [22], and block iterative support detection (blockISD) [14] have been shown to recover block sparse signals. In this paper, the BOMP algorithm [11] is implemented for the recovery of the block sparse channel vector.

BOMP algorithm is an extension of the orthogonal matching pursuit (OMP) algorithm [23, 24] used in conventional sparse recovery. The steps of the BOMP algorithm are shown in Algorithm 1.

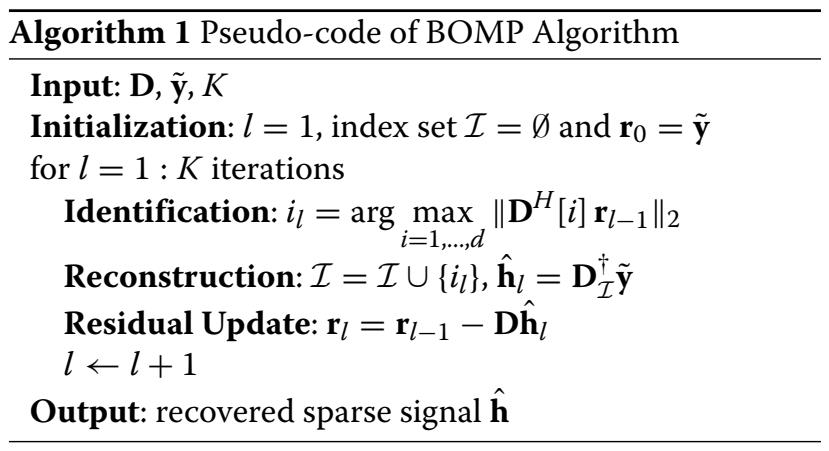

First step of the BOMP is to initialize the residual $\mathbf{r}_{0}=\tilde{\mathbf{y}}$ [11]. At the $l$ th iteration the block that is best matched to $\mathbf{r}_{l-1}$ is chosen according to

$$
i_{l}=\arg \max _{i=1, \ldots, d}\left\|\mathbf{D}^{H}[i] \mathbf{r}_{l-1}\right\|_{2} \text {. }
$$

Once the index $i_{l}$ is chosen, the CFO estimate $\hat{\epsilon}=\tilde{\epsilon}_{i_{l}}$ is the value corresponding to the chosen index within the search grid $\left\{\tilde{\epsilon}_{0}, \tilde{\epsilon}_{1}, \ldots, \tilde{\epsilon}_{d-1}\right\}$. The channel estimate $\hat{\mathbf{h}}_{l}$ is computed as

$$
\begin{aligned}
\hat{\mathbf{h}}_{l} & =\arg \min \left\|\tilde{\mathbf{y}}-\sum_{i \in \mathcal{I}} \mathbf{D}[i] \tilde{\mathbf{h}}_{l}[i]\right\|_{2} \\
& =\mathbf{D}_{\mathcal{I}}^{\dagger} \tilde{\mathbf{y}}=\left(\mathbf{D}_{\mathcal{I}}^{H} \mathbf{D}_{\mathcal{I}}\right)^{-1} \mathbf{D}_{\mathcal{I}}^{H} \tilde{\mathbf{y}},
\end{aligned}
$$

where $\mathcal{I}$ is the set of indices $\mathcal{I}=\left\{i_{1}, \ldots, i_{l}\right\}$ chosen up to iteration $l$. Then the residual is updated as

$$
\mathbf{r}_{l}=\tilde{\mathbf{y}}-\mathbf{D} \hat{\mathbf{h}}_{l} \text {. }
$$

For 1-block sparsity, the BOMP algorithm runs only $l=1$ iteration. The computational complexity of the algorithm for 1-block sparse signals is $\mathrm{O}\left(d N N_{g}\right)$. The BOMP method [11] selects the block that is most correlated with the current residual and then applies LS. So at any given iteration, $l$, of the BOMP method, the solution is guaranteed to be $l$ block-sparse. The performance of the BOMP method is shown to be better than the performance of the BIRLS method when the block sparsity is small [22]. BIRLS method [22] applies weighted LS in each iteration and depends on the weight matrix to make the solution sparse. In the first iteration of BIRLS method, the weight matrix is initialized to the identity matrix which means a regular LS is applied. In [14], block-ISD algorithm is implemented to estimate the CIR of a MIMO OFDM system with multiple transmit antennas and no CFO. Block sparse equivalent CIR is generated by assuming that the CIRs from different antennas share a common support. As it can be seen from Eq. (11), the block sparsity structure of our method is exploited by concatenating matrices corresponding to different frequency offsets and so does not use the common support assumption. The proposed dictionary (11) allows the joint estimation of both CFO and CIR while the dictionary generation shown in [14] does not allow the estimation of CFO. Block-ISD method [14] is based on the iterative support detection (ISD) reconstruction algorithm [25]. Like the matching pursuit methods, i.e., OMP [23, 24], a support set containing the locations of the detected nonzero elements is maintained in each iteration of the ISD method. At each iteration, this support set is used to solve a truncated basis pursuit (BP) problem by $\ell_{1}$ minimization. Unlike OMP, the support set of the ISD method can be updated with more than one elements in a given iteration.

The BOMP algorithm is shown to recover any $K$-block sparse vector $\tilde{\mathbf{h}}$ if the following condition is satisfied [11]

$$
K b<\left(\mu_{B}^{-1}+b-(b-1) v \mu_{B}^{-1}\right) / 2,
$$

where $b$ represents the block length for $\mathbf{D}$ and $\mu_{B}$ denotes the block-coherence

$$
\mu_{B}=\max _{l, r \neq l} \frac{1}{N_{g}} \sigma_{\max }\left(\mathbf{D}[l]^{H} \mathbf{D}[r]\right),
$$

and $v$ represents the subcoherence of $\mathbf{D}$

$$
v=\max _{i} \mu_{i}, \quad \mu=\max _{l, r \neq l}\left|\mathbf{d}_{l}^{H} \mathbf{d}_{r}\right|, \quad \mathbf{d}_{l}, \mathbf{d}_{r} \in \mathbf{D}[l] .
$$

If the columns of $\mathbf{D}[l]$ are orthonormal for each $l$, then the subcoherence is zero. Since the dictionary of the OFDM system consists of orthonormal blocks that are products of orthonormal matrices $\mathbf{D}[l]=\mathbf{C}\left(\tilde{\epsilon}_{l}\right) \mathbf{X F}_{N_{g}}, v=$ 0 . According to Eq. (16), the block length of 1-block sparse OFDM system, which is the cyclic prefix length $b=N_{g}$, must satisfy

$$
N_{g} \leq \frac{1}{\mu_{B}} .
$$




\section{Perturbation analysis for sparse multipath channel}

A $K$-sparse multipath channel is a channel of which the channel coefficient vector $\mathbf{h}$ has $K$ nonzero coefficients while the rest of $N_{g}-K$ tap values are equal to zero. For such a channel, the case of no frequency offset can be expressed as

$$
\tilde{\mathbf{y}}=\mathbf{y}+\mathbf{z}=\mathbf{A h}+\mathbf{z}=\mathbf{X F}_{N_{g}} \mathbf{h}+\mathbf{z}
$$

CS theory deals with the recovery of the signal $\mathbf{h}$ given the observations $\tilde{\mathbf{y}}$ using the a priori information that the signal $\mathbf{h}$ is $K$-sparse in the representation dictionary or matrix $\mathbf{A}$. In CS theory, the matrix $\mathbf{A}$ is called the sensing matrix for the $K$-sparse signal that is the channel coefficient vector $\mathbf{h}$. The stability of the CS based recovery methods depend on a property called the restricted isometry property (RIP). The RIP for the matrix $\mathbf{A}$ is defined as the smallest nonnegative number called the restricted isometry constant (RIC), $\delta_{K}$, for each integer $K=1,2, \ldots$ such that

$$
\left(1-\delta_{K}\right)\|\mathbf{h}\|_{2}^{2} \leq\|\mathbf{A h}\|_{2}^{2} \leq\left(1+\delta_{K}\right)\|\mathbf{h}\|_{2}^{2}
$$

holds for any $K$-sparse vector $\mathbf{h}$. It is observed that $\|\mathbf{A}\|_{2}^{(K)}=\sigma_{\max }^{(K)}(\mathbf{A}) \leq \sqrt{1+\delta_{K}}$ and $\sigma_{\min }^{(K)}(\mathbf{A}) \geq \sqrt{1-\delta_{K}}$, where $\|\mathbf{A}\|_{2}^{(K)}$ denotes the largest spectral norm, the largest singular value, taken over all $K$-column submatrices of $\mathbf{A}$ while $\sigma_{\min }^{K}(\mathbf{A})$ denotes the smallest nonzero singular value over all $K$-column submatrices of $\mathbf{A}$.

For the case of nonzero frequency offset, system of equations is given as

$$
\tilde{\mathbf{y}}=\mathbf{C}(\epsilon) \mathbf{X F}_{N_{g}} \mathbf{h}+\mathbf{z}=\tilde{\mathbf{A}} \mathbf{h}+\mathbf{z}
$$

The sensing matrix for this case, $\tilde{\mathbf{A}}=\mathbf{C}(\epsilon) \mathbf{X F}_{N_{g}}$, is a perturbed sensing matrix that can be written in terms of the sensing matrix for no frequency offset as

$$
\tilde{\mathbf{A}}=\mathbf{A}+\mathbf{Z}
$$

When (23) is substituted in (22), the received signal can be written as

$$
\tilde{\mathbf{y}}=\tilde{\mathbf{A}} \mathbf{h}+\mathbf{z}=(\mathbf{A}+\mathbf{Z}) \mathbf{h}+\mathbf{z}=\mathbf{A} \mathbf{h}+\mathbf{Z} \mathbf{h}+\mathbf{z}
$$

the extra noise term, $\mathbf{Z h}$, can be interpreted as multiplicative noise. The perturbation matrix $\mathbf{Z}$ can be explicitly expressed as

$$
\mathbf{Z}=\tilde{\mathbf{A}}-\mathbf{A}=\mathbf{C}(\epsilon) \mathbf{X F}_{N_{g}}-\mathbf{X F}_{N_{g}}=(\mathbf{C}(\epsilon)-\mathbf{I}) \mathbf{X F}_{N_{g}} .
$$

The total perturbation term, $\mathbf{Z h}+\mathbf{z}$, can be bounded by using a total noise parameter, $\varepsilon_{\mathbf{A}, K, \mathbf{y}}^{\prime}$, as in [26]

$$
\begin{aligned}
\|\mathbf{Z h}\|_{2}+\|\mathbf{z}\|_{2} & =\frac{\|\mathbf{Z} \mathbf{h}\|_{2}+\|\mathbf{z}\|_{2}}{\|\mathbf{y}\|_{2}}\|\mathbf{y}\|_{2}=\left(\frac{\|\mathbf{Z h}\|_{2}}{\|\mathbf{A} \mathbf{h}\|_{2}}+\frac{\|\mathbf{z}\|_{2}}{\|\mathbf{y}\|_{2}}\right)\|\mathbf{y}\|_{2} \\
& \leq\left(\frac{\|\mathbf{Z}\|_{2}^{(K)}}{\sqrt{1-\delta_{K}}} \frac{\|\mathbf{h}\|_{2}}{\|\mathbf{h}\|_{2}}+\varepsilon_{\mathbf{y}}\right)+\|\mathbf{y}\|_{2} \\
& \leq\left(\frac{\|\mathbf{Z}\|_{2}^{(K)}}{\sqrt{1+\delta_{K}}} \frac{\sqrt{1+\delta_{K}}}{\sqrt{1-\delta_{K}}}+\varepsilon_{\mathbf{y}}\right)+\|\mathbf{y}\|_{2} \\
& \leq\left(\frac{\|\mathbf{Z}\|_{2}^{(K)}}{\|\mathbf{A}\|_{2}^{(K)}} \kappa_{\mathbf{A}}^{(K)}+\varepsilon_{\mathbf{y}}\right)+\|\mathbf{y}\|_{2} \\
& \leq\left(\varepsilon_{\mathbf{A}}^{(K)} \kappa_{\mathbf{A}}^{(K)}+\varepsilon_{\mathbf{y}}\right)+\|\mathbf{y}\|_{2}=\varepsilon_{\mathbf{A}, K, \mathbf{y}}^{\prime},
\end{aligned}
$$

where $\mathbf{A}$ is assumed to satisfy RIP (21), $\|\mathbf{A}\|_{2}^{(K)} \leq$ $\sqrt{1+\delta_{K}}$, and the following relative bounds are used $\|\mathbf{z}\|_{2} /\|\mathbf{y}\|_{2} \leq \varepsilon_{\mathbf{y}},\|\mathbf{Z}\|_{2}^{(K)} /\|\mathbf{A}\|_{2}^{(K)} \leq \varepsilon_{\mathbf{A}}^{(K)} \cdot \kappa_{\mathbf{A}}^{(K)}$ is defined as the ratio of $\kappa_{\mathrm{A}}^{(K)}=\sqrt{\left(1+\delta_{K}\right) /\left(1-\delta_{K}\right)}$. For sensing matrices with very small $\delta_{K}$ which means that every $K$ column submatrice is approximately orthonormal, then $\kappa_{\mathrm{A}}^{(K)} \approx 1$. In the completely perturbed scenario (24) where $\mathbf{z} \neq 0$ and $\mathbf{Z} \neq 0$, CS theory is concerned with the solution of the $\mathrm{BP}$ problem

$$
\mathbf{h}^{\star}=\arg \min _{\hat{\mathbf{h}}}\|\hat{\mathbf{h}}\|_{1} \quad \text { subject to } \quad\|\tilde{\mathbf{A}} \hat{\mathbf{h}}-\tilde{\mathbf{y}}\| \leq \varepsilon_{\mathbf{A}, K, \mathbf{y}}^{\prime} .
$$

For the perturbed sensing matrix, $\tilde{\mathbf{A}}=\mathbf{A}+\mathbf{Z}$, of the completely perturbed problem (27), the RIC, $\hat{\delta}_{K}$, is defined as the smallest nonnegative number such that

$$
\left(1-\hat{\delta}_{K}\right)\|\mathbf{h}\|_{2}^{2} \leq\|\tilde{\mathbf{A}} \mathbf{h}\|_{2}^{2} \leq\left(1+\hat{\delta}_{K}\right)\|\mathbf{h}\|_{2}^{2}
$$

holds for any $K=1,2, \ldots$ sparse vector $\mathbf{h}$ [26]. The relation of the RIC $\hat{\delta}_{K}$ for $\tilde{\mathbf{A}}$ to the RIC $\delta_{K}$ for $\mathbf{A}(21)$ and the relative perturbation $\varepsilon_{\mathbf{A}}^{(K)}$ is shown in [26] as

$$
\hat{\delta}_{K} \leq\left(1+\delta_{K}\right)\left(1+\varepsilon_{\mathbf{A}}^{(K)}\right)^{2}-1 .
$$

Let $\mathbf{e}$ denote the perturbation from the true solution $\mathbf{h}$ of the BP problem (27) induced by $\mathbf{Z}$ and $\mathbf{z}$. Then, the minimizer of the BP problem can be given as $\mathbf{h}^{\star}=\mathbf{h}+\mathbf{e}$. Using the steps in [27], the perturbation from the true solution, $\mathbf{e}$, can be decomposed into a sum of vectors $\mathbf{e}_{S_{0}}$ and $\mathbf{e}_{S_{1}}$

$$
\begin{aligned}
\left\|\mathbf{h}^{\star}-\mathbf{h}\right\|_{2} & =\|\mathbf{e}\|_{2} \leq\left\|\mathbf{e}_{\left(S_{0} \cup S_{1}\right)}\right\|_{2}+\| \mathbf{e}_{\left(S_{0} \cup S_{1}\right)^{c} \|_{2}} \\
& \leq \frac{4 \sqrt{\left(1+\hat{\delta}_{2 K}\right)}}{1-(1+\sqrt{2}) \hat{\delta}_{2 K}} \varepsilon_{\mathbf{A}, K, \mathbf{y}}^{\prime}
\end{aligned}
$$

where $S_{0}$ corresponds to the locations of the $K$ largest coefficients of $\mathbf{h}$, and $S_{1}$ corresponds to the locations of the $K$ largest coefficients of $\mathbf{e}_{0}^{c}$. Using (29) and 
substituting $2 K$ in place of $K$, it is shown in [26] that the solution of the completely perturbed problem (27) is bounded by

$$
\begin{aligned}
\left\|\mathbf{h}^{\star}-\mathbf{h}\right\|_{2} \leq & \frac{4 \sqrt{1+\delta_{2 K}}\left(1+\varepsilon_{\mathbf{A}}^{(2 K)}\right)}{1-(\sqrt{2}+1)\left[\left(1+\delta_{2 K}\right)\left(1+\varepsilon_{\mathbf{A}}^{(2 K)}\right)^{2}-1\right]} \\
& \times \varepsilon_{\mathbf{A}, K, \mathbf{y}}^{\prime}=C_{1} \varepsilon_{\mathbf{A}, K, \mathbf{y}}^{\prime},
\end{aligned}
$$

provided that the sensing matrix A satisfies the RIP property (21) for

$$
\delta_{2 K}<\frac{\sqrt{2}}{\left(1+\varepsilon_{\mathbf{A}}^{(2 K)}\right)^{2}}-1 .
$$

In order for A to satisfy Eq. (32), the RIC must be nonnegative $0<\delta_{2 K}$ and so the relative perturbation must be less than $\varepsilon_{\mathbf{A}}^{(2 K)}<\sqrt[4]{2}-1 \approx 0.1892$.

The upper bound given in (31) can be considered the worst instance of the worst-case scenarios. CS recovery methods are known to achieve much better performance in practice [26]. A lower bound or the best of the worstcase scenarios can be analyzed if the support set $S$ of $\mathbf{h}$, the locations of the $K$ nonzero coefficients, is provided by an oracle. Let $\mathbf{A}_{S}$ denote the submatrix consisting of the columns of $\mathbf{A}$ indexed by the elements of the support set $S$. Given the completely perturbed observation, the leastsquares problem of the channel estimation

$$
\mathbf{h}_{S}^{\#}=\arg \min \left\|\tilde{\mathbf{A}}_{S} \mathbf{h}_{S}-\tilde{\mathbf{y}}\right\|_{2} .
$$

Golub and Van Loan's model in Theorem 5.3.1 of [28] formulates the perturbed LS problem by subtituting $\tilde{\mathbf{A}}_{S}=$ $\mathbf{A}_{S}+(t / \varepsilon) \mathbf{Z}_{S}$ and $\tilde{\mathbf{y}}=\mathbf{y}+(t / \varepsilon) \mathbf{z}$ as

$$
\left(\mathbf{A}_{S}+\frac{t}{\varepsilon} \mathbf{Z}_{S}\right)^{H}\left(\mathbf{A}_{S}+\frac{t}{\varepsilon} \mathbf{Z}_{S}\right) \mathbf{h}_{S}(t)=\left(\mathbf{A}_{S}+\frac{t}{\varepsilon} \mathbf{Z}_{S}\right)^{H}\left(\mathbf{y}+\frac{t}{\varepsilon} \mathbf{z}\right)
$$

where the solution is assumed to be continuously differentiable for all $t \in[0, \varepsilon]$. With the solution given as $\mathbf{h}_{S}^{\#}=$ $\mathbf{h}_{S}+\varepsilon \dot{\mathbf{h}}_{S}(0)+O\left(\varepsilon^{2}\right)[28]$, the error can be bounded as

$$
\left\|\mathbf{h}_{S}^{\#}-\mathbf{h}_{S}\right\|_{2}=\varepsilon\left\|\dot{\mathbf{h}}_{S}(0)\right\|_{2}+O\left(\varepsilon^{2}\right) .
$$

Differentiating Eq. (34) and setting $t=0$ yields

$$
\dot{\mathbf{h}}_{S}(0)=\frac{1}{\varepsilon}\left(\mathbf{A}_{S}^{H} \mathbf{A}_{S}\right)^{-1} \mathbf{A}_{S}^{H}\left(\mathbf{z}-\mathbf{Z}_{S} \mathbf{h}_{S}\right) .
$$

The solution is bounded above by inserting (36) into (35)

$$
\left\|\mathbf{h}^{\#}-\mathbf{h}\right\|_{2} \leq\left\|\mathbf{A}_{S}^{\dagger}\right\|_{2}\left(\frac{\left\|\mathbf{Z}_{S} \mathbf{h}_{S}\right\|_{2}}{\|\mathbf{A} \mathbf{h}\|_{2}}+\varepsilon_{\mathbf{y}}\right)\|\mathbf{y}\|_{2},
$$

where the complete solution, $\mathbf{h}^{\#}$, is obtained by extending $\mathbf{h}_{S}^{\#}$ with zero-padding on the complement of the support set $S$ and $\mathbf{A}_{S}^{\dagger}=\left(\mathbf{A}_{S}^{H} \mathbf{A}_{S}\right)^{-1} \mathbf{A}_{S}^{H}$ is the pseudoinverse of $\mathbf{A}_{S}[26]$.

\section{Numerical results}

Numerical results are presented for three scenarios: timeinvariant sparse multipath channel, time-varying sparse multipath channel, and time-varying sparse multipath with varying CFO. The number of carriers of the OFDM system is chosen as $N=128$. The length of the sparse multipath channel is fixed to $L=20$ with $K=6$ nonzero coefficients for all cases. The nonzero coefficients of the channel are generated from independent complex Gaussian with variances set according to an exponential decaying power delay profile. The CP length is set to $N_{g}=25$ to prevent IBI. The mean squared error of the frequency estimates (MSE CFO) and channel estimates (MSE CIR) are given for 1000 Monte Carlo iterations. The pilots symbols are randomly chosen from 16-QAM constellation and then are fixed for each Monte Carlo iteration.

\subsection{Time-invariant sparse multipath channel}

The sparse multipath channel is assumed to be fixed between two consecutive OFDM blocks. The magnitude of the generated sparse multipath channel is shown in Fig. 1 , and it can be seen that only $K=6$ coefficients of the CIR are nonzero.

MSE obtained using the BOMP algorithm is compared to pilot symbol-based Moose [1], Classen [2] methods, the approximate MLE [9], and the p-algorithm [5] for CFO value $\epsilon=0.03$. The search space of the BOMP, the approximate MLE, and the p-algorithm is assigned to the set of 500 uniformly sampled values within the interval

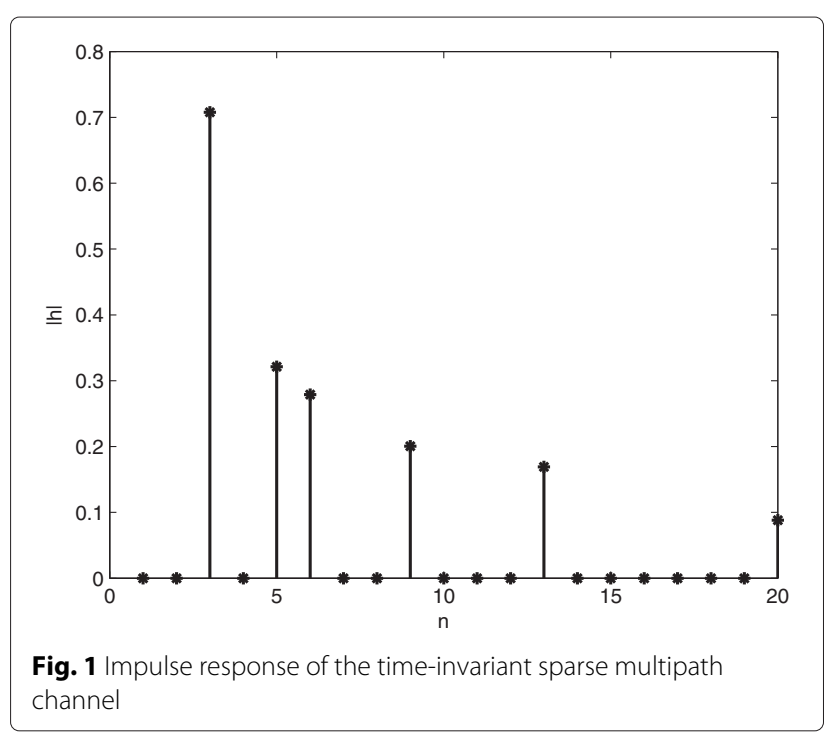


[ $-0.5,0.5]$. The actual CFO value, $\epsilon=0.03$, is included in the search grid to prevent error floors in the MSE of the CFO estimates. The block coherence of the dictionary built using the search grid $\mu_{B}(\mathbf{D})=0.04$ satisfy the recovery condition given in Eq. (19) for $N_{g}=25$. MSE of the CFO estimates are illustrated in Fig. 2. Moose and Classen methods perform better than the BOMP and the MLE for SNR levels below $15 \mathrm{~dB}$. Moose and Classen methods ignore the ICI and so when the SNR is low, ignoring the ICI term does not degrade the performance significantly. However, as the SNR increases beyond 15 $\mathrm{dB}$, the Moose and Classen methods reach an error floor. The performance of the BOMP algorithm is close to the performance of the MLE which is implemented by using the actual channel and noise statistics. Since in practice it will also be neccessary to estimate both the channel and noise statistics, the performance of the MLE given in Fig. 2 can be considered an ideal case. It is seen that the best performance is achieved by the p-algorithm [5]. Although the p-algorithm makes use of two consecutive OFDM blocks like the Moose and Classen methods, it is based on cost function minimization using a search space and so it performs better than the Moose and Classen methods. The Cramer-Rao bound (CRB) (Fig. 2) given in [29] represents a lower bound for the maximum likelihood estimation using one OFDM block of pilot symbols. CRB is used as a benchmark for evaluating different CFO estimators and the closest performance to CRB belongs to the p-algorithm.

Figure 3 shows the MSE of CIR estimates. The performance of the least-squares (LS) CIR estimate can be improved by first applying a CFO synchronization method. LS method combined with the p-algorithm can be considered a two step joint CFO and CIR estimator.

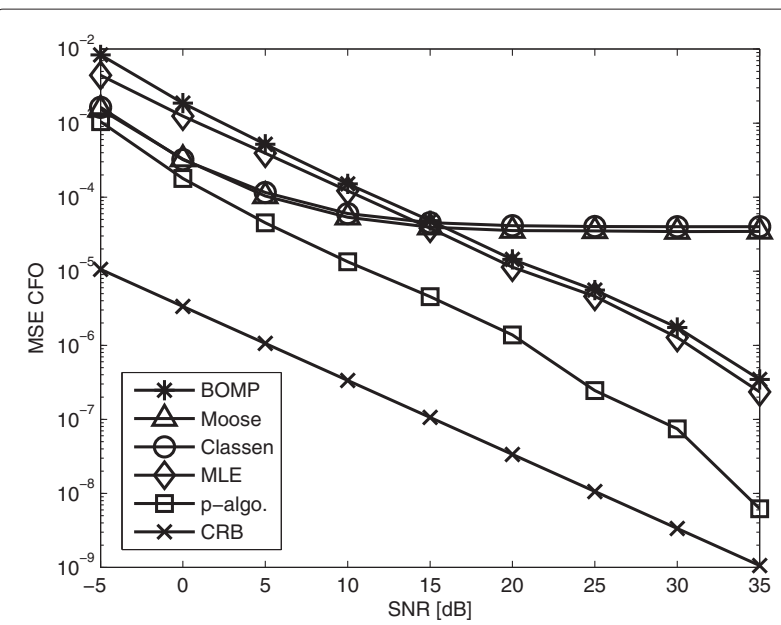

Fig. 2 Comparison of MSE of CFO estimates versus SNR for the time invariant sparse channel with $\epsilon=0.03$

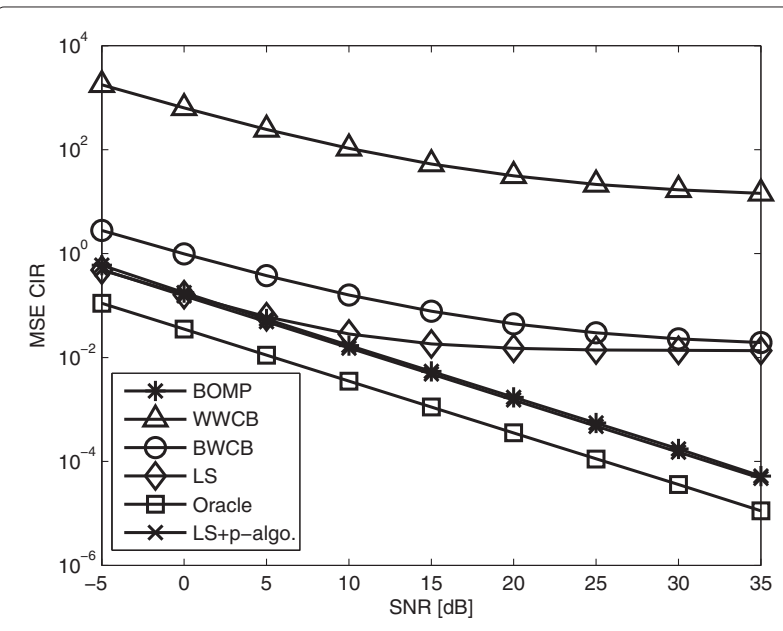

Fig. 3 Comparison of MSE of CIR estimates versus SNR for the time invariant sparse channel with $\epsilon=0.03$

Figure 3 indicates that the two step LS estimator gives the best performance and the BOMP method performs closely to the two step LS estimator. The best possible channel estimate is represented by the oracle which is computed using the actual CFO value and the support of the channel. The closest performance to the oracle is achieved by the two step LS estimator and the BOMP method. Using the perturbation analysis (Section 4), two upper bounds for the channel estimation performance of the OFDM system with $\mathrm{CFO} \epsilon=0.03$ can be obtained. These bounds are plotted as WWCB and BWCB (Fig. 3) which stands for worst worst-case upper bound given in Eq. (31) and best worst-case upper bound given in Eq. (37), respectively. The coefficients for the WWCB are computed as $\varepsilon_{\mathbf{A}}^{(K)}=0.1545, \varepsilon_{\mathbf{A}}^{(2 K)}=0.1601, C_{1}=25.06$ and $\kappa_{\mathrm{A}}^{(K)}=1$. Since the exact computation of the RIC $\delta_{K}$ and $\delta_{2 K}$ is not possible, $\sigma_{\max }^{(K)}(\mathbf{A})$ and $\sigma_{\min }^{(K)}(\mathbf{A})$ are computed to obtain a bound for the RIC. The bound obtained is on the order of $10^{-15}$ making the RIC practically zero. WWCB shows the worst possible channel estimation performance for a OFDM system with CFO $\epsilon=0.03$ and a sparse multipath channel with $K=6$ nonzero coefficients. BWCB gives a much tighter bound than WWCB since it makes use of the channel tap values in its computations. The advantage of WWCB is that its computation only requires the sparsity level of the multipath channel.

In order to demonstrate that the performance of the proposed joint estimator does not depend on the CFO value, the SNR is fixed at $15 \mathrm{~dB}$ and the CFO is varied in a set of 30 uniformly spaced values within the interval $[-0.5,0.5]$. As the value of CFO increases, MSE of the Moose and Classen methods increase due to their sensitivity to the value of the CFO while MSE of the BOMP method does not increase as seen in Fig. 4. Classen's 


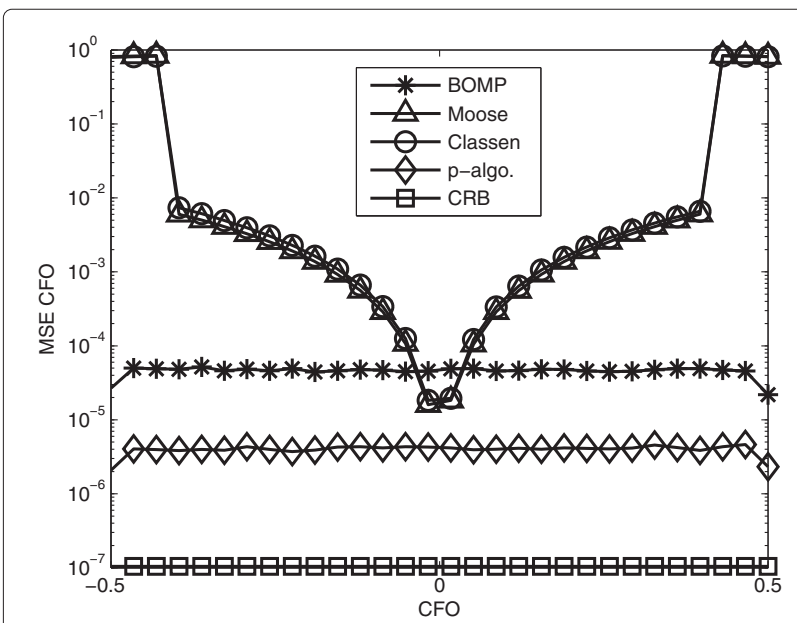

Fig. 4 Comparison of MSE of CFO estimates versus CFO range of $[-0.5,0.5]$ for the time-invariant sparse channel at $15 \mathrm{~dB}$ SNR

method assumes a sufficiently small CFO in order for ICI to be neglected so the performance of the Classen's method degrades as the CFO increases. Only for very small CFO values, $\epsilon \in[-0.0172,0.0172]$, the performances of the Moose and Classen methods are better than the BOMP method. The BOMP method and the palgorithm do not exhibit any sensitivity to the value of the CFO and their performances do not depend on the value of the CFO. The p-algorithm gives the closest performance to the CRB but needs at least two OFDM blocks like the Moose and Classen methods.

MSE of CIR estimates are given first for a smaller CFO range of $[-0.03,0.03]$ as seen in Fig. 5. WWCB cannot be computed for $\epsilon>0.0306$ since the relative bound on the perturbation must satisfy $\varepsilon_{\mathrm{A}}^{(2 K)}<0.1892$ and so the CFO range is assigned to the set of 12 uniformly sampled values

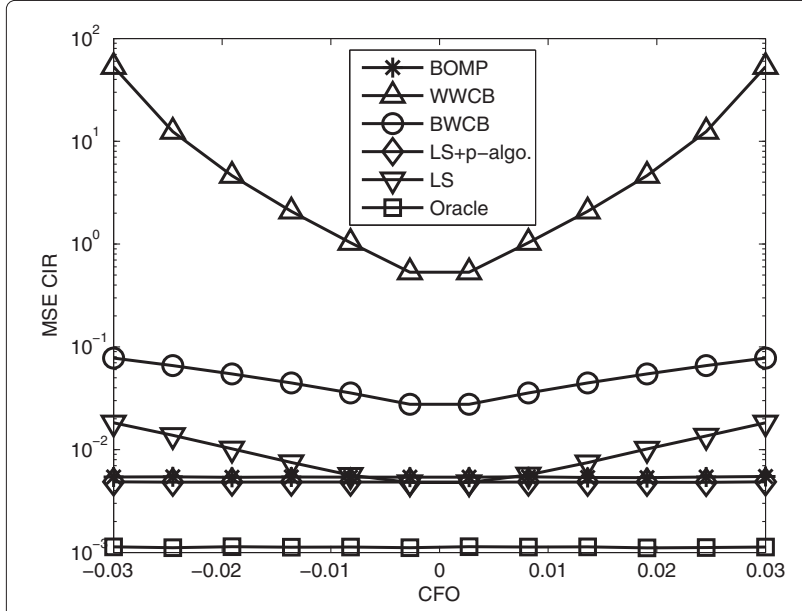

Fig. 5 Comparison of MSE of CIR estimators versus CFO range of $[-0.03,0.03]$ for the time-invariant sparse channel at $S N R=15 \mathrm{~dB}$ within the interval [ $-0.03,0.03]$. For this range of smaller CFO values, it is observed that the WWCB becomes much looser as the CFO increases. Thus the WWCB can be considered much more sensitive to $C F O$ value than the BWCB. The best performance is achieved by the two-step LS estimator, while the performance of the BOMP method is close. As expected, the oracle bound is the lower bound for the sparse channel estimation and is not subject to any change by CFO value since it is provided with the actual CFO value and support of the sparse channel.

Figure 6 gives the MSE of CIR estimates versus the extended range of $\mathrm{CFO}$ values, $[-0.5,0.5]$. Of the two upper bounds, only the BWCB can be shown for the CIR estimate within this range since it is not possible to calculate the WWCB for $\epsilon>0.0306$. Although the BWCB is a much tighter bound and can be computed for the full CFO range, the computation of the $\mathrm{BWCB}$ requires the prior knowledge of the channel. On the other hand, the computation of the WWCB does not require the channel information. Best performance is achieved by the two-step LS estimator combined with the p-algorithm while the proposed BOMP is very close.

\subsection{Time-varying sparse multipath channel}

Depending on the mobility of the receiver, the channel may remain essentially constant over the duration of the block, or may be slowly time varying. For time-varying channels, the CIR cannot be assumed to be static over two consecutive OFDM blocks.

\subsubsection{Correlated time-varying sparse multipath channel}

When the channel is time varying, the nonzero channel coefficients may vary slowly and are temporally correlated. The parameters of the channel may remain constant over several OFDM blocks. Consequently, the locations

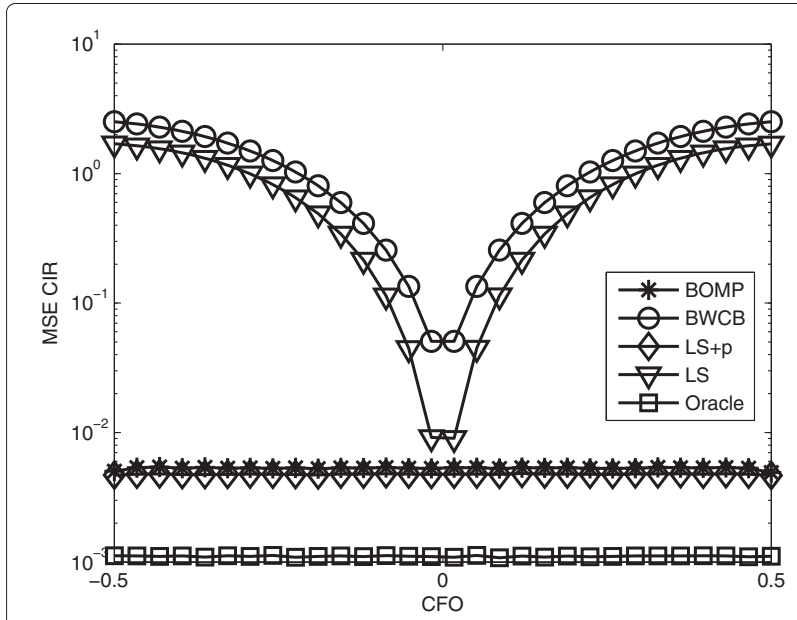

Fig. 6 Comparison of MSE of CIR estimates versus CFO range of $[-0.5,0.5]$ for the time-invariant sparse channel at $S N R=15 \mathrm{~dB}$ 
of the nonzero channel coefficients coincide in successive channel instantiations. The temporal variations of such a channel can be captured by an autoregressive (AR) state space model. It is shown in that the local behaviour of fading wireless channels is accurately represented by the first order AR model [30]. The temporally correlated timevarying sparse channel is generated using a first-order AR model given as

$$
\mathbf{h}_{m+1}=\rho \mathbf{h}_{m}+\mathbf{u}_{m} \quad m=1,2, \ldots
$$

where $\mathbf{u}_{m}$ denotes the driving noise and $\rho$ is calculated using the zeroth order Bessel function of the first kind, $\rho=J_{0}(2 \pi \Delta f T)$ [15]. Each entry of the driving noise is generated independently from zero mean complex white Gaussian distribution with variance $\left(1-\rho^{2}\right) \gamma(l)[15,31]$. The sparse channel is initialized using multivariate complex white Gaussian distribution with covariance matrix $\Gamma=\operatorname{diag}(\gamma(1), \ldots, \gamma(L))$ where variances $\gamma(l)$ are set according to the same exponential power decay profile used in Section 5.1. MSE of CFO estimates for the correlated time carying sparse channel are shown in Fig. 7. For $\mathrm{SNR}=-5 \mathrm{~dB}$ the $\mathrm{p}$-algorithm yields lower MSE than the MLE and the BOMP methods. As SNR increases beyond $-5 \mathrm{~dB}$, the performance of the the p-algorithm stays the same. The performances of the Moose, Classen and the palgorithm cannot be improved with increasing SNR due to the time varying channel. The p-algorithm performs better than the Moose and Classen methods. The performances of the MLE and the BOMP methods are not affected by the time-varying channel.

Figure 8 shows the MSE of CIR estimates for the correlated time-varying channel. The pure LS estimator without any CFO synchronization performs closely to the BOMP method for SNR below $10 \mathrm{~dB}$. As SNR increases beyond $10 \mathrm{~dB}$, the MSE of the pure LS estimator reaches

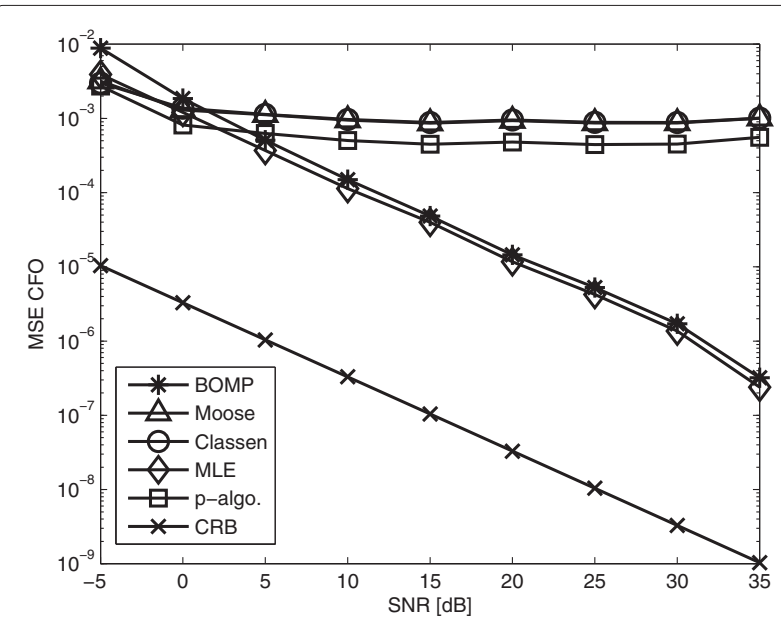

Fig. 7 Comparison of MSE of CFO estimates versus SNR for correlated time-varying sparse channel with $\epsilon=0.03$

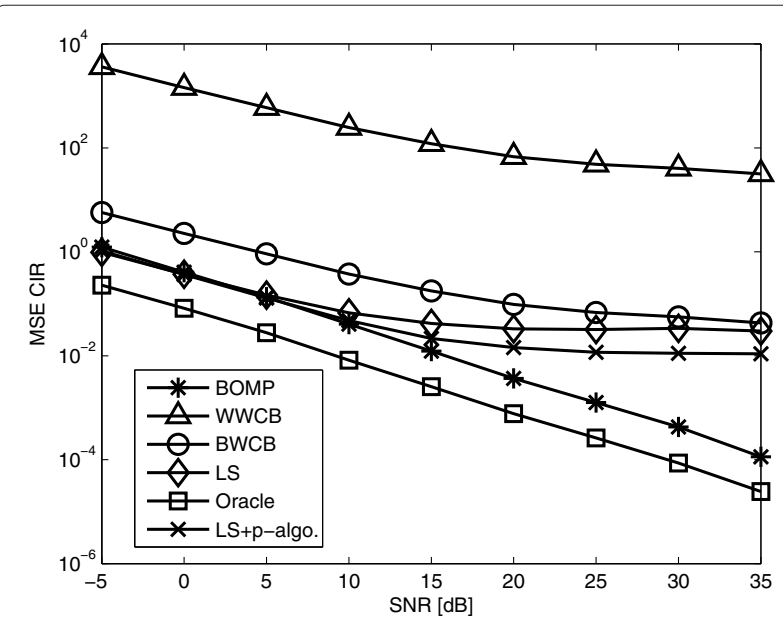

Fig. 8 Comparison of MSE of CIR estimates versus SNR for correlated time varying sparse channel with $\epsilon=0.03$

an error floor seen in Fig. 8. As in Fig. 3, the BWCB becomes much tighter bound as the SNR increases for the time varying sparse channel. The upper bounds WWCB and BWCB exhibit an error floor as the SNR increases for the time-varying sparse channel. Degradation due to the time-varying channel is not observed for the BOMP method since the BOMP method does not use a channel model.

MSE of CFO estimates versus the CFO range of $[-0.5,0.5]$ are presented in Fig. 9. The p-algorithm no longer holds the performance advantage but manages to perform better than the Moose and Classen methods. As the CFO increases, the performance of the Moose and Classen methods degrades severely. Figure 9 shows that the Moose and Classen methods are prone to greater performance degradation for larger CFO values when the sparse channel is time varying. The $\mathrm{p}$-algorithm does not show sensitivity to $C F O$ value.

WWCB for the correlated time varying sparse channel is illustrated in Fig. 10. When the sparse channel is time varying, it is seen that the performance the pure LS method can perform better than the two-step LS estimator combined with the p-algorithm for smaller CFO values. MSE of CIR estimates versus the extended CFO range $[-0.5,0.5]$ are presented in Fig. 11. For smaller CFO values, the pure LS channel estimate is better than the two-step LS estimate using the p-algorithm synchronization.

\subsubsection{Uncorrelated time-varying sparse multipath channel}

The support, the nonzero taps, of the sparse multipath channel are generated from uniform distribution while the nonzero coefficients are generated from standard Gaussian distribution for each OFDM block. MSE of CFO estimates for this case are shown in Fig. 12. The 


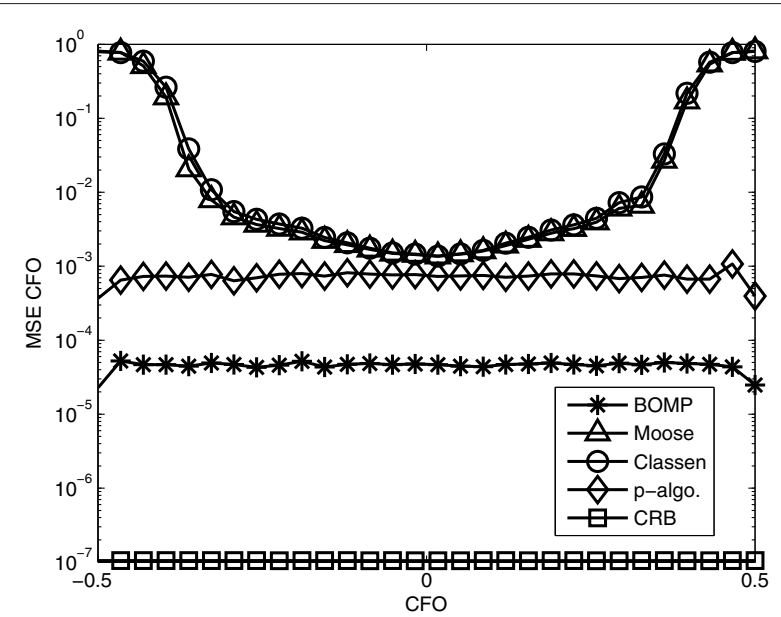

Fig. 9 Comparison of MSE of CFO estimates versus CFO range of $[-0.5,0.5]$ for correlated time varying sparse channel at $S N R=15 \mathrm{~dB}$

performances of the Moose, Classen, and the p-algorithm does not improve as the SNR increases if the sparse channel changes between two consecutive OFDM blocks. All of the three methods which rely on consecutive OFDM blocks, yield the worst performance. The performances of the MLE and the BOMP methods stay consistent with the performances given for the time invariant channel since they use only one OFDM block and are not affected by the changing channel.

Figure 13 shows the MSE of CIR estimates for the timevarying channel. It is observed that the performance of the two-step LS estimator using the p-algorithm is worse than the pure LS estimate which does not use any CFO synchronization. The performance of the two-step LS estimator is observed to be even worse than the BWCB for SNR greater than $0 \mathrm{~dB}$. The pure LS estimator without any CFO

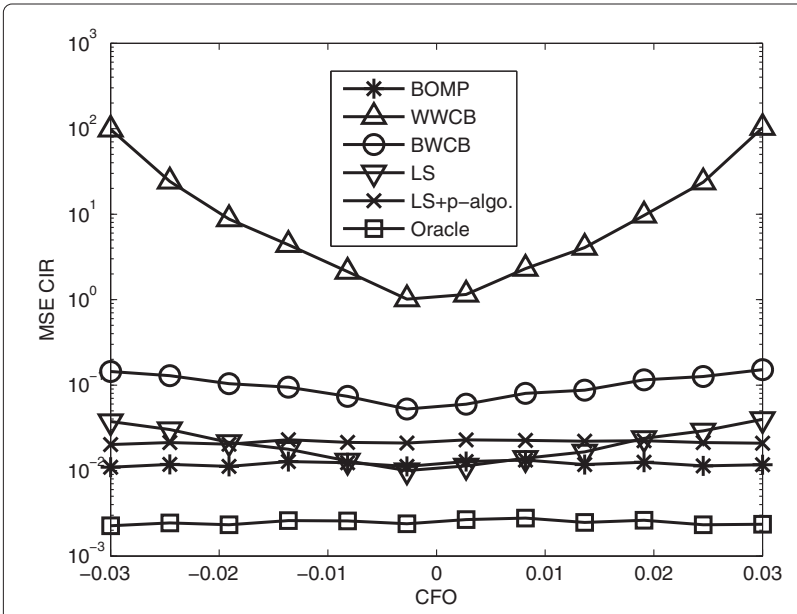

Fig. 10 Comparison of MSE of CIR estimates versus CFO range of $[-0.03,0.03]$ for correlated time-varying sparse channel at $15 \mathrm{~dB}$ SNR

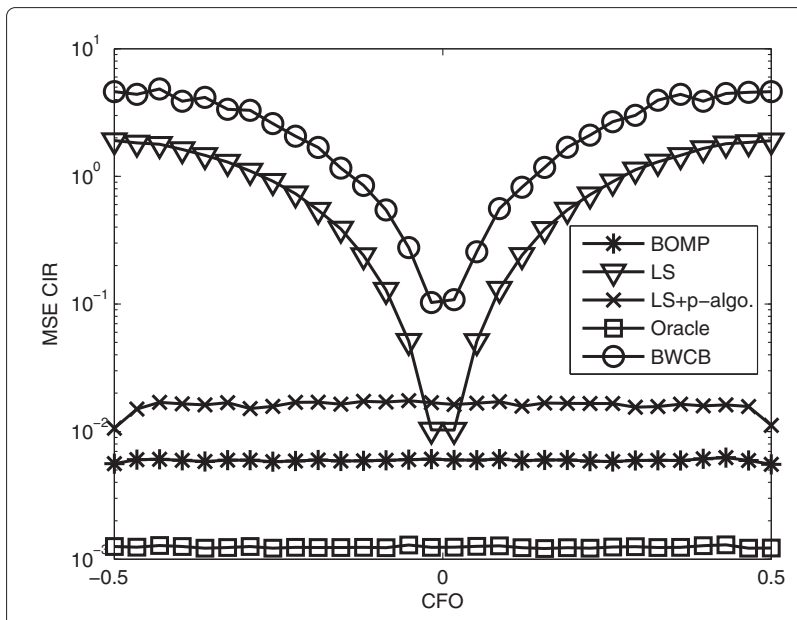

Fig. 11 Comparison of MSE of CIR estimates versus CFO range of $[-0.5,0.5]$ for correlated time-varying sparse channel at $15 \mathrm{~dB}$ SNR

synchronization performs closely to the BOMP method for SNR below $10 \mathrm{~dB}$. As SNR increases beyond $10 \mathrm{~dB}$, the MSE of the pure LS estimator reaches an error floor as seen in Fig. 13. Figure 13 indicates that the upper bounds WWCB and BWCB exhibit an error floor as the SNR increases for the time-varying sparse channel. Figure 13 shows that the performance of the BOMP method does not exhibit any degradation for the time varying channel as a consequence of not relying on a channel model. The BOMP method gives the closest performance to the oracle bound for the time-varying sparse channel. MSE of CFO estimates versus the CFO range of $[-0.5,0.5]$ are presented in Fig. 14. The Moose, Classen, and the palgorithm give the worst performance as seen in Fig. 14 . The closest performance to the CRB bound is achieved by the BOMP method.

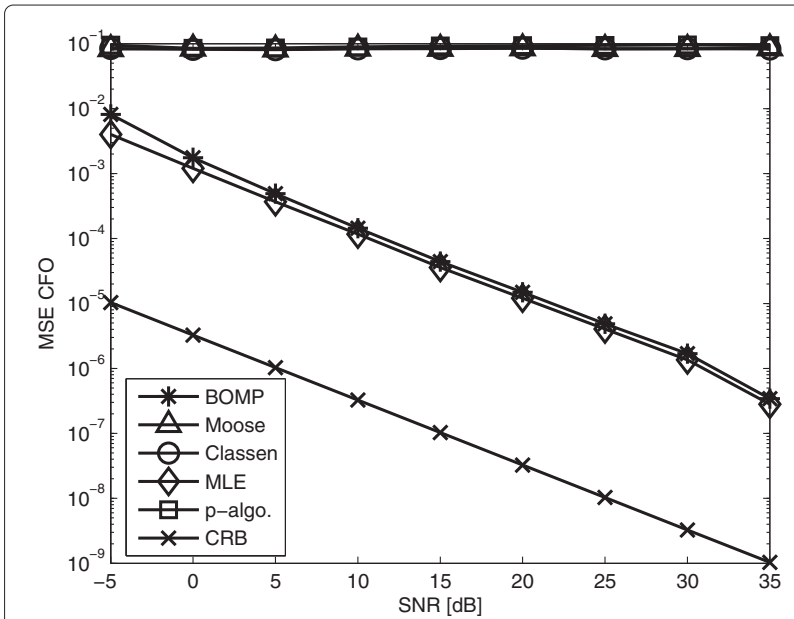

Fig. 12 Comparison of MSE of CFO estimates versus SNR for uncorrelated time-varying sparse channel with $\epsilon=0.03$ 


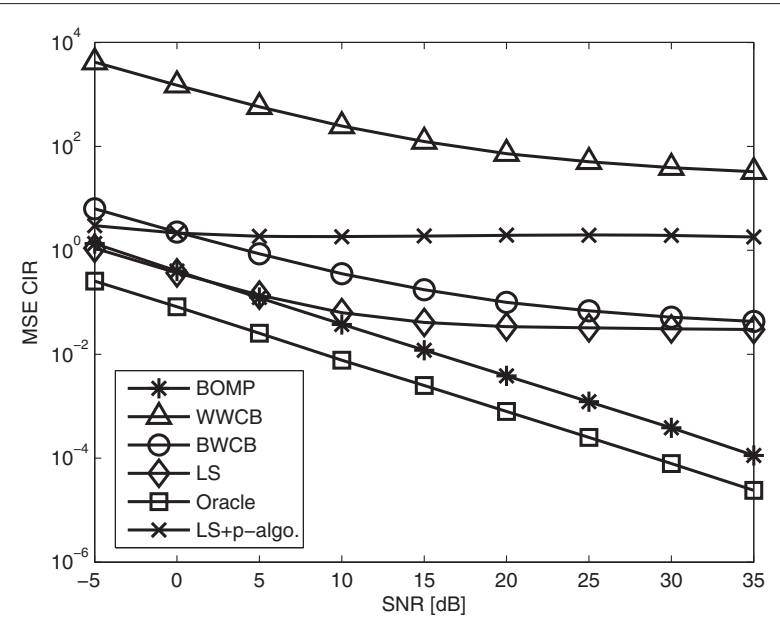

Fig. 13 Comparison of MSE of CIR estimates versus SNR for uncorrelated time-varying sparse channel with $\epsilon=0.03$

WWCB for the time-varying sparse channel is illustrated in Fig. 15. When the sparse channel is time varying, it is seen that the performance the pure LS method is much better than the performance of the two-step LS estimator combined with the p-algorithm. MSE of CIR estimates versus the extended CFO range $[-0.5,0.5]$ are presented in Fig. 16. For smaller CFO values the pure LS channel estimate yields lower MSE than the two-step LS estimate using the $\mathrm{p}$-algorithm synchronization. The closest performance to the oracle bound versus the whole CFO range is achieved by the BOMP method.

\subsection{Uncorrelated time-varying sparse multipath channel with varying CFO}

CFO may also be subject to change between each successive OFDM block. In order to give numerical results

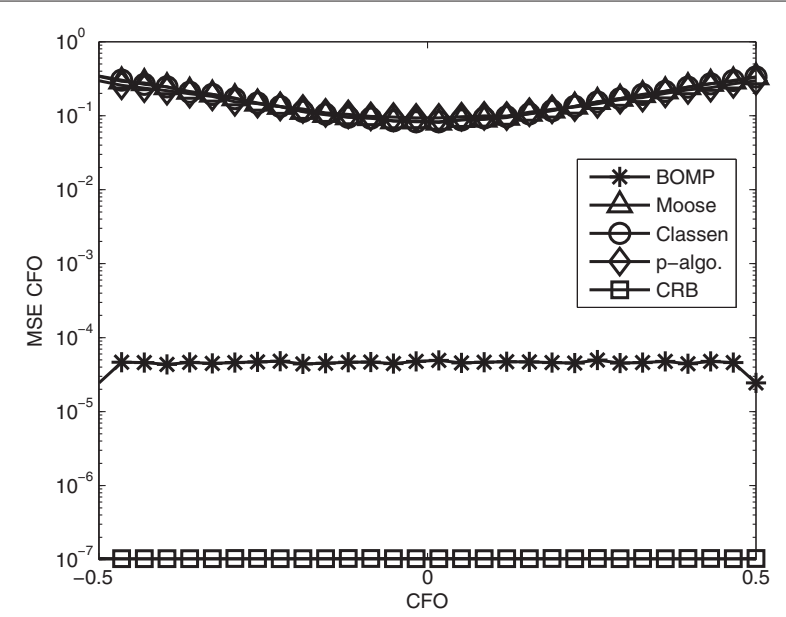

Fig. 14 Comparison of MSE of CFO estimates versus CFO range of $[-0.5,0.5]$ for uncorrelated time-varying sparse channel at SNR $=15 \mathrm{~dB}$

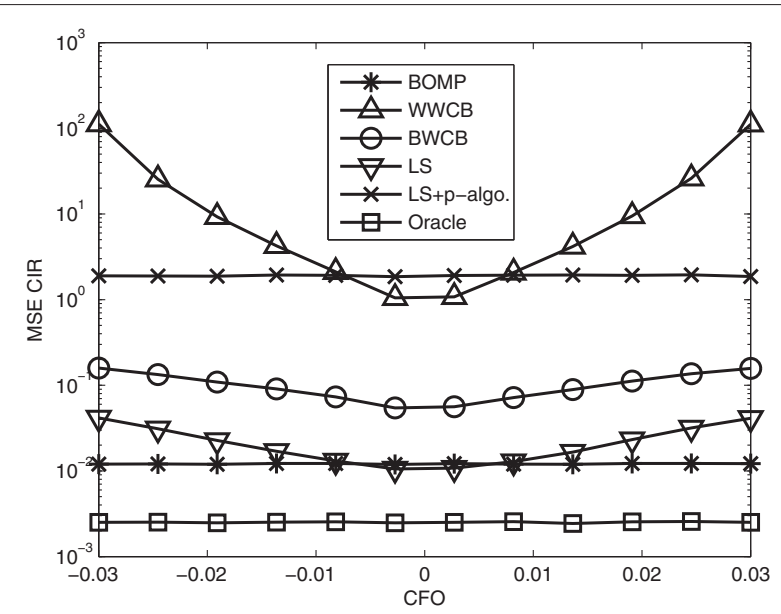

Fig. 15 Comparison of MSE of CIR estimates versus CFO range of $[-0.03,0.03]$ for uncorrelated time-varying sparse channel at $15 \mathrm{~dB}$ SNR

for this case, $\mathrm{CFO}$ is assumed to be uniformly distributed in the interval $\epsilon \sim U(-0.03,0.03)$ for each successive OFDM block as in [29]. The sparse multipath channel is time varying as in Section 5.2. Figure 17 gives the MSE of CFO estimates versus SNR for varying channel and CFO. The Moose, Classen, and p-algorithm perform worse than the MLE and the BOMP methods since both CFO and CIR is changing for each OFDM block. The BOMP and the MLE methods give the same performance and are not affected by the changing CFO and CIR. Figure 18 shows the MSE of CIR estimates. The pure LS method and the LS method combined with the CFO estimate provided by the p-algorithm produces the same MSE. They are subject to the same error floor as the BWCB for SNR levels above $25 \mathrm{~dB}$. The closest performance to the oracle estimator is achieved by the BOMP method.

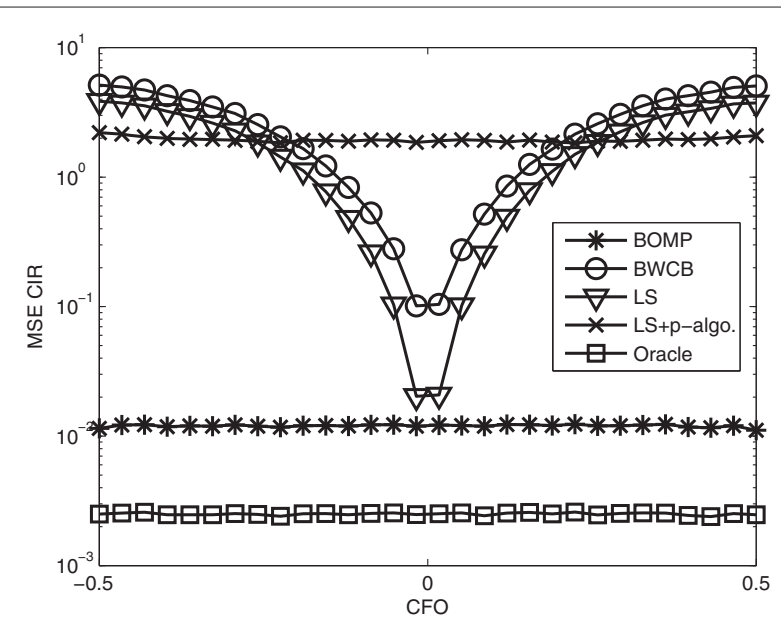

Fig. 16 Comparison of MSE of CIR estimates versus CFO range of $[-0.5,0.5]$ for uncorrelated time-varying sparse channel at $15 \mathrm{~dB}$ SNR 


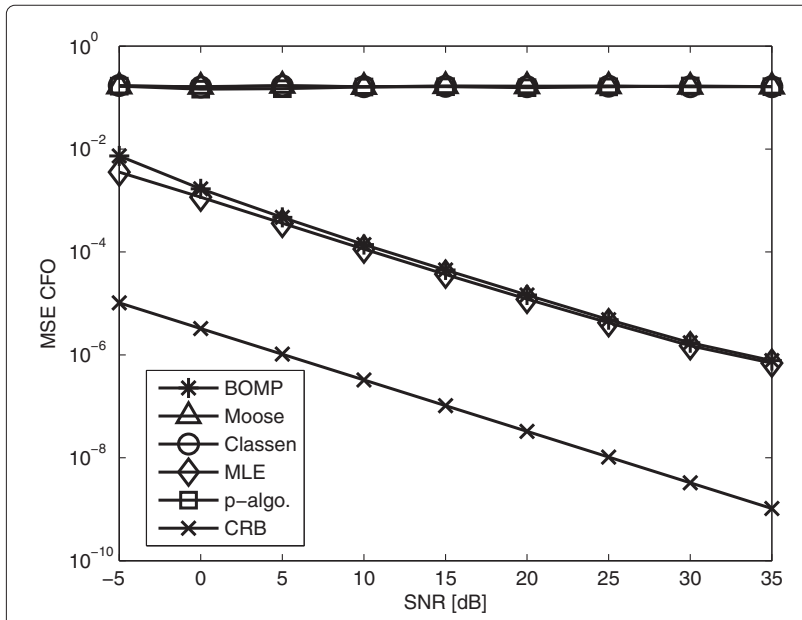

Fig. 17 Comparison of MSE of CFO estimates versus SNR for time-varying sparse channel with $\epsilon \sim U(-0.03,0.03)$

\section{Conclusions}

We introduced a novel CS based framework for the joint estimation of CFO and CIR in OFDM systems. It is shown that the CIR can be represented as a 1-block sparse signal if a dictionary is built by concatenating subspaces of CFO values within a search grid. CS theory allows the recovery of signals that are given in such representation. The BOMP algorithm is used to reconstruct the CIR coefficients. The proposed estimator uses only one block of training symbols and no initialization is needed. Worst case analysis using perturbation bounds from CS theory are applied to sparse channel estimation. Numerical results show that the proposed estimator gives the same performance as the MLE.

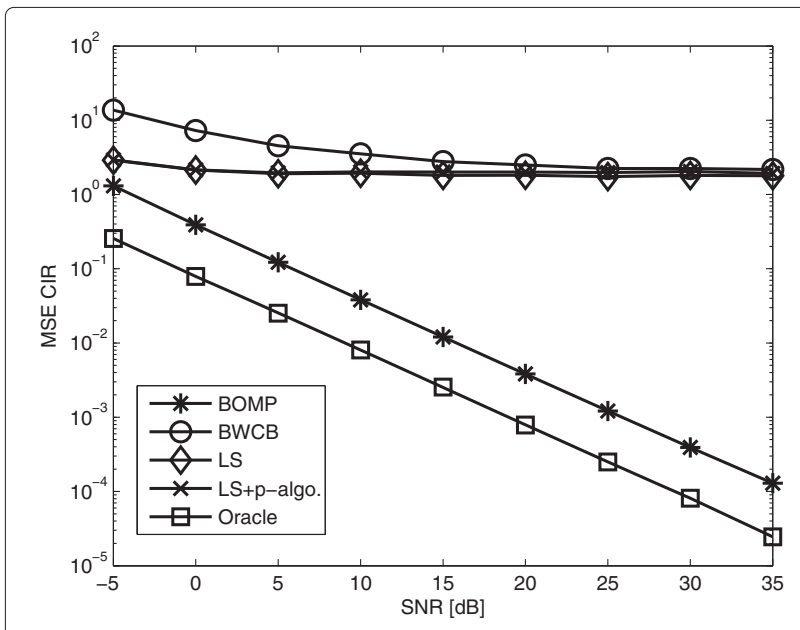

Fig. 18 Comparison of MSE of CIR estimates versus SNR for time-varying sparse channel with $\epsilon \sim U(-0.03,0.03)$

\section{Competing interests}

The authors declare that they have no competing interests.

\section{Author details}

'Department of Electrical and Electronics Engineering, Bogazici University, Bebek, Istanbul, Turkey. ${ }^{2}$ Department of Electronics and Communication Engineering, Istanbul Technical University, Maslak, Istanbul, Turkey.

Received: 3 June 2015 Accepted: 3 March 2016

Published online: 22 March 2016

\section{References}

1. PH Moose, A technique for orthogonal frequency division multiplexing frequency offset correction. IEEE Trans. Comm. 42(10), 2908-2914 (1994)

2. F Classen, H Meyr, in Vehicular Technology Conference, 1994 IEEE 44th, Stockholm. Frequency synchronization algorithms for OFDM systems suitable for communication over frequency selective fading channels, vol. 3, (1994), pp. 1655-1659. doi:10.1109/NETEC.1994.345377, http:// ieeexplore.ieee.org/stamp/stamp.jsp?tp=\&arnumber= 345377\&isnumber $=8028$

3. J-J Van de Beek, M Sandell, PO Borjesson, ML estimation of time and frequency offset in OFDM systems. IEEE Trans. Signal Proc. 45(7), 1800-1805 (1997)

4. H Liu, U Tureli, A high-efficiency carrier estimator for OFDM communications. IEEE Comm. Lett. 2(4), 104-106 (1998)

5. F Gao, T Cui, A Nallanathan, Scattered pilots and virtual carriers based frequency offset tracking for OFDM systems: algorithms, identifiability, and performance analysis. IEEE Trans. Comm. 56(4), 619-629 (2008)

6. J-H Lee, JC Han, S-C Kim, Joint carrier frequency synchronization and channel estimation for OFDM systems via the EM algorithm. IEEE Trans. Veh. Tech. 55(1), 167-172 (2006)

7. EP Simon, L Ros, H Hijazi, M Ghogho, Joint carrier frequency offset and channel estimation for OFDM systems via the EM algorithm in the presence of very high mobility. IEEE Trans. Signal Proc. 60(2), 754-765 (2012)

8. M Morelli, U Mengali, Carrier-frequency estimation for transmissions over selective channels. IEEE Trans. Comm. 48(9), 1580-1589 (2000)

9. T Cui, C Tellambura, Joint frequency offset and channel estimation for OFDM systems using pilot symbols and virtual carriers. IEEE Trans. Wireless Comm. 6(4), 1193-1202 (2007)

10. DL Donoho, Compressed sensing. IEEE Trans. Info. Theory. 52(4), 1289-1306 (2006)

11. YC Eldar, P Kuppinger, H Bolcskei, Block-sparse signals: uncertainty relations and efficient recovery. IEEE Trans. Signal Proc. 58(6), 3042-3054 (2010)

12. E Elhamifar, R Vidal, Block-sparse recovery via convex optimization. IEEE Trans. Signal Proc. 60(8), 4094-4107 (2012)

13. Y Barbotin, A Hormati, S Rangan, M Vetterli, Estimation of sparse MIMO channels with common support. IEEE Trans. Comm. 60(12), 3705-3716 (2012)

14. W Shen, L Dai, Z Gao, Z Wang, Spatially correlated channel estimation based on block iterative support detection for massive MIMO systems. Electron. Lett. 51(7), 587-588 (2015)

15. R Prasad, CR Murthy, BD Rao, Joint approximately sparse channel estimation and data detection in OFDM systems using sparse Bayesian learning. IEEE Trans. Signal Proc. 62(14), 3591-3603 (2014)

16. J Lee, H-L Lou, D Toumpakaris, JM Cioffi, SNR analysis of OFDM systems in the presence of carrier frequency offset for fading channels. IEEE Trans. Wireless Comm. 5(12), 3360-3364 (2006)

17. TM Schmidl, DC Cox, Robust frequency and timing synchronization for OFDM. IEEE Trans. Comm. 45(12), 1613-1621 (1997)

18. M Morelli, AN D'Andrea, U Mengali, Frequency ambiguity resolution in OFDM systems. IEEE Comm. Lett. 4(4), 134-136 (2000)

19. M Yuan, Y Lin, Model selection and estimation in regression with grouped variables. J. R. Statist. Soc. B. 68(1), 49-67 (2006)

20. YC Eldar, M Mishali, Robust recovery of signals from a structured union of subspaces. IEEE Trans. Info. Theory. 55(11), 5302-5316 (2009)

21. Z Zhang, BD Rao, in Acoustics, Speech and Signal Processing (ICASSP), 2012 IEEE International Conference on. Recovery of block sparse signals using the framework of block sparse Bayesian learning, (Kyoto, 2012), 
pp. 3345-3348. doi:10.1109/ICASSP.2012.6288632, http://ieeexplore.ieee. org/stamp/stamp.jsp?tp=\&arnumber=6288632\&isnumber $=6287775$

22. $\mathrm{SLi}, \mathrm{Q} \mathrm{Li}, \mathrm{G} \mathrm{Li}, \mathrm{X} \mathrm{He}, \mathrm{L}$ Chang, in Industrial Electronics and Applications (ICIEA), 2014 IEEE 9th Conference on. Iteratively reweighted least squares for block-sparse recovery, (Hangzhou, 2014), pp. 1061-1066.

doi:10.1109/ICIEA.2014.6931321, http://ieeexplore.ieee.org/stamp/stamp. jsp?tp=\&arnumber $=6931321$ \&isnumber $=6931119$

23. S Mallat, Z Zhang, Matching pursuits with time-frequency dictionaries. IEEE Trans. Signal Proc. 41(12), 3397-3415 (1993)

24. JA Tropp, AC Gilbert, Signal recovery from random measurements via orthogonal matching pursuit. IEEE Trans. Info. Theory. 53(12), 4655-4666 (2007)

25. Y Wang, W Yin, Sparse signal reconstruction via iterative support detection. SIAM J. Imaging Sci. 3(3), 462-491 (2010)

26. MA Herman, T Strohmer, General deviants: an analysis of perturbations in compressed sensing. IEEE Sig. Proc. Sel. Top. 4(2), 342-349 (2010)

27. EJ Candès, The restricted isometry property and its implications for compressed sensing. Comptes Rendus Mathematique. 346(9-10), 589-592 (2008)

28. GH Golub, CF Van Loan, Matrix Computations (3rd edn.) (Johns Hopkins University Press, Baltimore, MD, USA, 1996)

29. L Bai, Q Yin, CRB for carrier frequency offset estimation with pilot and virtual subcarriers. IEEE Comm. Lett. 16(4), 522-525 (2012)

30. TFeng, TR Field, S Haykin, Stochastic differential equation theory applied to wireless channels. IEEE Trans. Comm. 55(8), 1478-1483 (2007)

31. W Chen, R Zhang, in Acoustics, Speech, and Signal Processing, 2004. Proceedings. (ICASSP '04). IEEE International Conference on. Kalman-filter channel estimator for OFDM systems in time and frequencyselective fading environment, vol. 4, (2004), pp. iv-377-iv-380. doi:10.1109/ICASSP.2004.1326842, http://ieeexplore.ieee.org/stamp/ stamp.jsp?tp=\&arnumber $=1326842 \&$ isnumber $=29346$

\section{Submit your manuscript to a SpringerOpen ${ }^{\circ}$ journal and benefit from:}

- Convenient online submission

$\checkmark$ Rigorous peer review

- Immediate publication on acceptance

- Open access: articles freely available online

- High visibility within the field

- Retaining the copyright to your article

Submit your next manuscript at $\gg$ springeropen.com 